\title{
Three-layered synthetic pericardial substitutes reduce postoperative pericardial adhesions
}

Noriyoshi Kajihara, $\mathrm{MD}^{\mathrm{a}}$

Masataka Eto, MD

Yasuhisa Oishi, MD

Noriko Boku, MD

Kunio Kuwahara, BS ${ }^{\text {b }}$

Naoki Nishiguchi, MS ${ }^{b}$

Chikara Kotanib

Shigeki Morita, $\mathrm{MD}^{\mathrm{a}}$

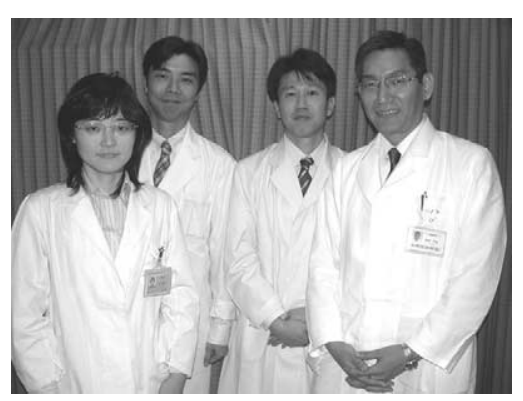

Background: The development of postoperative pericardial adhesions increases the risk of cardiac reoperations. The purpose of this study was to test a new pericardial substitute (UBE sheet; UBE Industries, Ltd, Tokyo, Japan) that consists of 3 layers, namely, a middle layer of polyester inserted between 2 layers of silicone-urethane copolymer.

Methods: Before implantation into the animals, platelet adhesion to the UBE sheet was evaluated in vitro. In the canine model, the UBE sheet (group I; $n=6$ ) was implanted for 3 months. The development of adhesions, epicardial reactions, the shrink ratio of the patch, and macrophage infiltration to the epicardium with histologic examination were evaluated. As a control, an expanded polytetrafluoroethylene sheet (group II; $n=5$ ) was implanted in the same manner.

Results: Scanning electron microscopy of the platelets adhered to the sheet showed that the UBE sheet was superior in biocompatibility compared with the expanded polytetrafluoroethylene sheet. In the canine study, group I showed fewer adhesions than group II (median [25th percentile, 75th percentile]: 0.0 [0.0, 0.0] vs 1.0 [1.0, 2.3]; $P=.003$; Mann-Whitney $U$ test), fewer epicardial reactions $(1.75[1.0,3.0]$ vs $3.0[3.0,3.0] ; P=.034)$, and a smaller shrink ratio $(8.0 \%[5.5 \%, 12.4 \%]$ vs $31.7 \%$ $[30.0 \%, 44.8 \%] ; P=.006)$. Immunohistologic studies showed fewer macrophage infiltrations $(86[56.8,139.3]$ vs $201[161.0,276.5]$ in 3 fields; $P=.045)$ into the epicardium of group I.

From the Department of Cardiovascular Surgery, Graduate School of Medical Sciences, Kyushu University, Fukuoka, Japan, a and Ube Industry Ltd, Ichihara, Japan. ${ }^{\text {b }}$

Received for publication Oct 30, 2003; revisions requested Feb 23, 2004; revisions received March 8, 2004; accepted for publication March 16, 2004.

Address for reprints: Shigeki Morita, MD, Department of Cardiovascular Surgery, Graduate School of Medical Sciences, Kyushu University, 3-1-1 Maidashi, Higashi$\mathrm{Ku}$, Fukuoka 812-8582, Japan (E-mail: morita@heart.med.kyushu-u.ac.jp).

J Thorac Cardiovasc Surg 2005;129:18-24

0022-5223/\$30.00

Copyright (C) 2005 by The American Association for Thoracic Surgery

doi:10.1016/j.jtcvs.2004.03.010

Conclusions: The new 3-layered pericardial substitute clearly reduced adhesion formation. We concluded that this sheet may cause fewer adhesions and a less severe inflammatory reaction after cardiac surgery, thereby facilitating safe adhesiolysis reoperation.

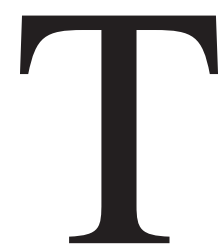

he development of postoperative pericardial adhesions increases the risk of cardiac reoperations. ${ }^{1,2}$ Closing the native pericardium after cardiac operation is the most popular way to reduce the risk of reoperation. However, pericardial closure is often not possible because of a previous cardiac operation or the risk of extracardiac graft compression. Therefore, many surgeons have used various biologic or synthetic sheets as a pericardial substitute. ${ }^{3-10}$ So far, however, there have been no adequate substitutes that reduce adhesions and epicardial reactions.

Polyether-based polyurethanes have been widely used as biomaterials because of their biocompatibility and their desirable physical properties, such as strength and flexibility. However, polyether polyurethanes containing polyether soft segments 

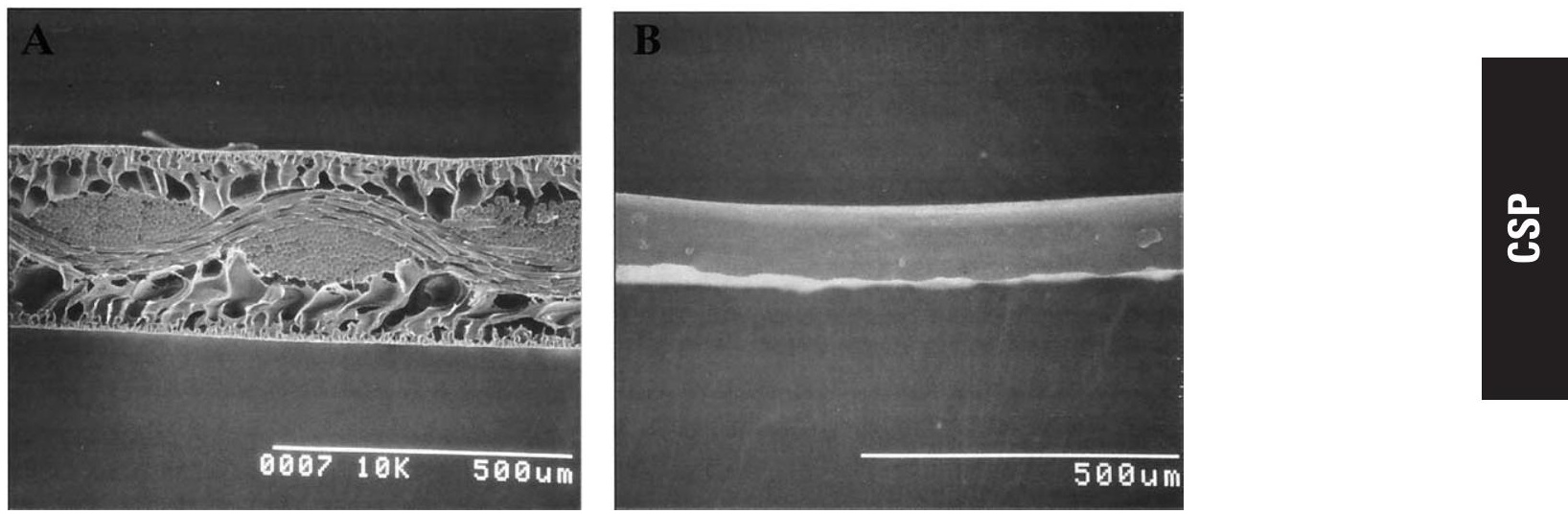

Figure 1. Scanning electron microscopic views of the cross sections of the sheet (original magnification, $\times 100$ ). $A$, The 3 layers of the UBE sheet: a middle layer of polyester is inserted between 2 layers of silicone-urethane copolymer. B, The ePTFE sheet, consisting of 1 layer.

are susceptible to oxidative cleavage in vivo. ${ }^{11,12}$ Modifications of the soft segment have been made to enhance biostability, ${ }^{13-15}$ and several studies have shown that the polycarbonate soft segment is more stable than the polyether soft segment. ${ }^{16,17}$ Furthermore, silicone-urethane copolymers that possess the biocompatibility and biostability of conventional silicone elastomers with the processability and toughness of thermoplastic polyurethanes were recently produced, and segmented polycarbonate urethanes have been shown to enhance biocompatibility and biostability via silicone copolymerization. ${ }^{18}$

The purpose of this study was to investigate whether a new synthetic pericardial substitute consisting of 3 layersnamely, a middle layer of polyester inserted between 2 layers of silicone-urethane copolymer-could attenuate the host reaction, thereby reducing adhesion and epicardial reaction. As a control, an expanded polytetrafluoroethylene (ePTFE) sheet was used.

\section{Methods}

\section{Manufacturing Process of the UBE Sheet}

The silicone-urethane copolymer (CarboSil-10; The Polymer Technology Group Inc, Berkeley, Calif) was dissolved with $\mathrm{N}, \mathrm{N}$ dimethyl formamide. A sheet made from polyethyleneterephtalate was fixed between stainless-steel boards $0.2 \mathrm{~mm}$ thick and with an area of $100 \times 100 \mathrm{~mm}$ cut into the central part. It was then immersed in a silicone-urethane copolymer solution. After excessive solution was removed, the sheet was immersed in distilled water at $20^{\circ} \mathrm{C}$ and finally washed with ethanol. The sheet (UBE sheet model CL-0202; UBE Industries, Ltd, Tokyo, Japan) consists of 3 layers, namely, a middle layer of polyester inserted between 2 layers of silicone-urethane copolymer (Figure 1,A).

\section{Platelet Adhesion Studies in Vitro}

Blood samples of mongrel dogs were collected in tubes containing $3.8 \%$ sodium citrate and centrifuged at $900 \mathrm{rpm}$ for 10 minutes to

\section{TABLE 1. The scoring system of macroscopic evaluation}

\begin{tabular}{|c|c|}
\hline \multicolumn{2}{|c|}{ Adhesion } \\
\hline 0 & No adhesion \\
\hline 1 & $\begin{array}{l}\text { Minimal adhesion; can be easily } \\
\text { separated with finger dissection }\end{array}$ \\
\hline 2 & $\begin{array}{l}\text { Moderate adhesion; aggressive } \\
\text { dissection required for separation }\end{array}$ \\
\hline 3 & $\begin{array}{l}\text { Severe adhesion; sharp dissection } \\
\text { required for separation }\end{array}$ \\
\hline \multicolumn{2}{|c|}{ Epicardial reaction } \\
\hline 0 & No fibrosis \\
\hline 1 & $\begin{array}{l}\text { Minimal fibrotic layer; the coronary } \\
\text { anatomy or other underlying } \\
\text { structures were easily identified }\end{array}$ \\
\hline 2 & $\begin{array}{l}\text { Moderate fibrotic layer; the recognition } \\
\text { of underlying structures was } \\
\text { severely impeded }\end{array}$ \\
\hline 3 & $\begin{array}{l}\text { Severe fibrotic layer, the underlying } \\
\text { structures were not identifiable }\end{array}$ \\
\hline
\end{tabular}

obtain platelet-rich plasma (PRP). The sheets were exposed to PRP for 10 or 40 minutes. The sheets were washed with modified Hanks solution and fixed by immersion in $2.5 \%$ glutaraldehyde solution, followed by critical-point drying. The specimens were examined with a scanning electron microscope (model S-510; Hitachi, Tokyo, Japan). The UBE sheet and ePTFE sheet (Gore-Tex pericardial membrane; W. L. Gore \& Associates, Inc, Flagstaff, Ariz) were compared with regard to the presence of platelet aggregations.

\section{Canine Study}

All animals received humane care in compliance with the "Principles of Laboratory Animal Care" formulated by the National Society for Medical Research and the "Guide for the Care and Use of Laboratory Animals" prepared by the National Institutes of Health publication No. 86-23, revised 1985). 

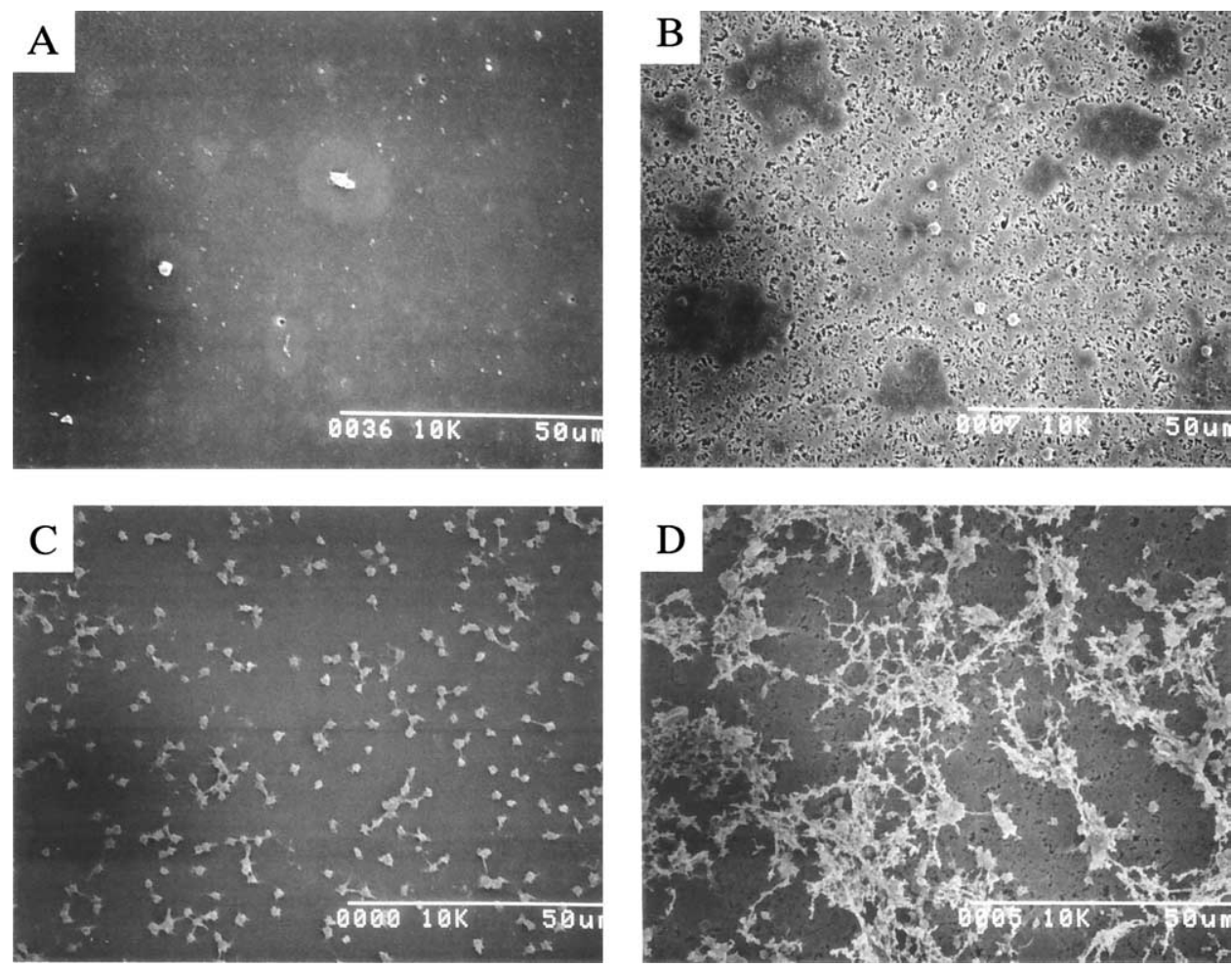

Figure 2. Scanning electron microscopic views of the adherent platelets on surfaces of the sheets. Platelet-rich plasma was put on the UBE sheet for 10 minutes (A) and 40 minutes (C) and on the ePTFE for 10 minutes (B) and 40 minutes (D).

TABLE 2. The results of the macroscopic evaluation of adhesions, epicardial reactions, and shrink ratios in the canine model

\begin{tabular}{lcccc}
\hline Group & $\begin{array}{c}\text { Adhesion to the } \\
\text { epicardium }\end{array}$ & $\begin{array}{c}\text { Adhesion to the } \\
\text { suture line }\end{array}$ & $\begin{array}{c}\text { Epicardial } \\
\text { reaction }\end{array}$ & Shrink ratio (\%) \\
\hline Group I & $0.0(0.0,0.0)$ & $0.0(0.0,1.3)$ & $1.8(1.0,3.0)$ & $8.0(5.5,12.4)$ \\
Group II & $1.0(1.0,2.3)$ & $2.0(2.0,2.5)$ & $3.0(3.0,3.0)$ & $31.7(30.0,44.8)$ \\
$P$ value & .003 & .011 & .034 & .006 \\
\hline
\end{tabular}

Values are median (25th, 75th percentile).

\section{Operative Procedure}

Eleven mongrel dogs weighing from 11.2 to $17.8 \mathrm{~kg}$ were divided into 2 groups: a UBE sheet group (group I; $\mathrm{n}=6$ ) and an ePTFE sheet group (group II; $n=5$ ). The dogs were anesthetized with sodium thiamylal $(10 \mathrm{mg} / \mathrm{kg})$ and intubated with a tracheal tube connected to a mechanical ventilator (model PLV-100; Respironics Inc, Westminster, Colo). Anesthesia was maintained with vecuronium bromide $(0.4 \mathrm{mg} / \mathrm{kg})$ and midazolam $(0.5 \mathrm{mg} / \mathrm{kg})$. A median sternotomy was performed by a standard aseptic technique. A piece of pericardium $50 \times 75 \mathrm{~mm}$ was excised, and the defect was repaired with the sheet. The animals were allowed to wake up on the operating table, after which they were extubated. Antibiotic therapy was maintained for 5 days after surgery.

Re-sternotomy was performed 3 months after the first operation. The shrink ratios [(pre-area - post-area $) /$ pre-area $\times 100]$ of the sheet with appearance just after re-sternotomy were evaluated.
Two observers evaluated the adhesion formation and epicardial reactions to the sheet (Table 1). The animals were killed with a lethal dose of anesthesia. Their hearts were removed and immersed in $20 \%$ paraformaldehyde for histopathologic examination.

\section{Histopathology and Immunohistochemistry}

Histologic specimens were fixed in $20 \%$ paraformaldehyde, embedded in paraffin, and cut into slices. Some of these sections were stained with hematoxylin and eosin and Azan-Mallory stains. The remaining sections were used for immunostaining. Sections were incubated for 60 minutes at room temperature with the mouse monoclonal anti-human macrophage/monocyte antibody MAC387 (Dako Cytomation Co Ltd, Copenhagen, Denmark). The number of macrophages in the epicardium was counted as the sum of the cell counts on 3 fields at $\times 400$ magnification. 

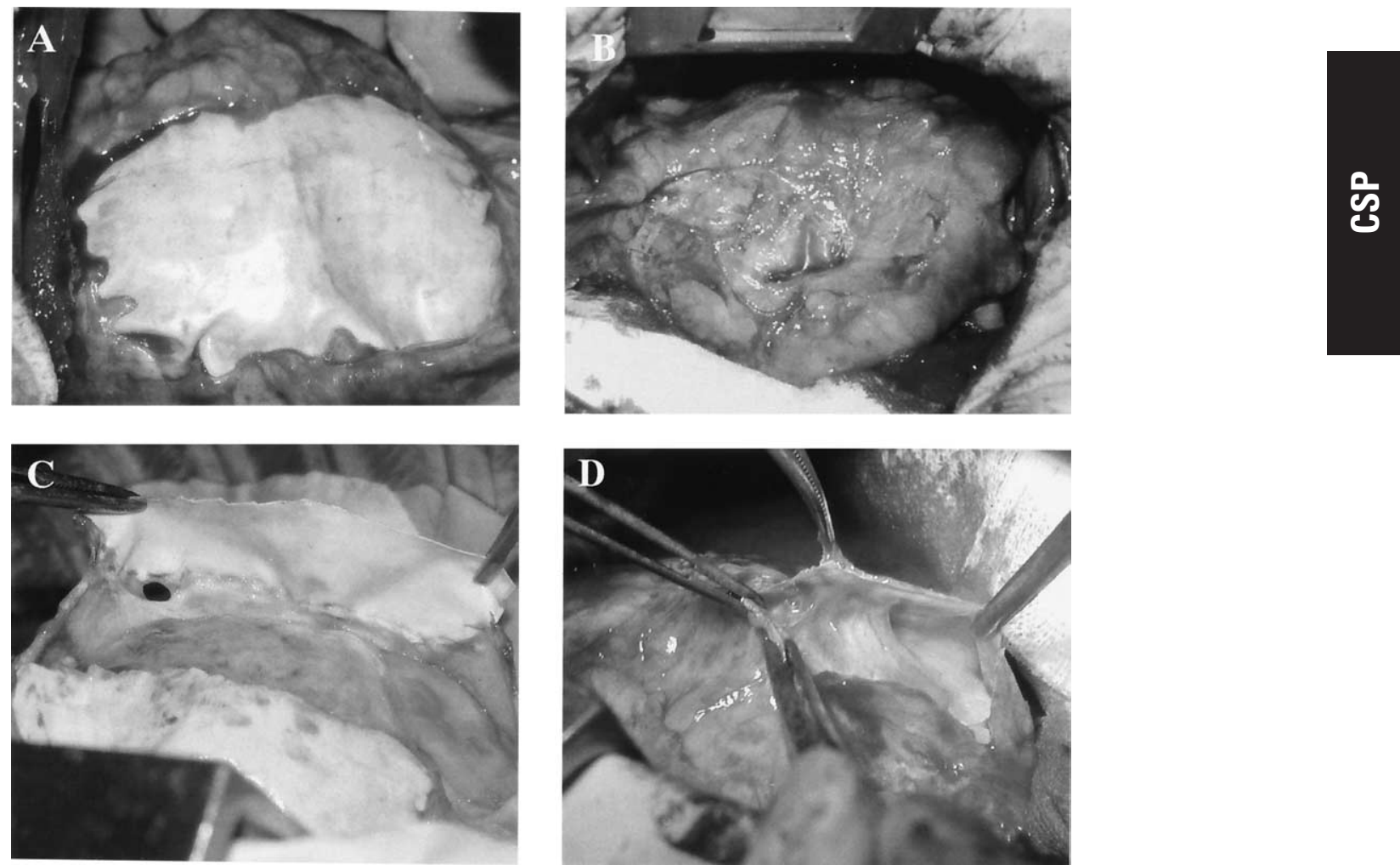

Figure 3. The appearances of the sheets 3 months after implantation. A, The sheets of group I retained the implanted form. B, The sheets of group II were crinkled, less pliable, and thickened by a dense layer of fibrous tissue. C, In group I, there were no adhesions to the sheets, including the suture line, and there were minimum epicardial reactions. D, In group II, there were moderate adhesions to the sheets and severe epicardial reactions.

\section{Statistical Analysis}

Analyses were performed with SPSS software (version $11.0 \mathrm{~J}$; SPSS Inc, Chicago, Ill). Variables are expressed as the median (25th percentile, 75 th percentile). Differences between experimental groups were determined with the Mann-Whitney $U$ test.

\section{Results}

Scanning Electron Microscopic Observations of the Adherent Platelets

Scanning electron microscopy of the adherent platelets to the UBE and ePTFE patches under static conditions is shown in Figure 2. A few individual platelets adhered to both of the sheets with a slight shape change after 10 minutes (Figure 2, $A$ and $B$ ). Many individual platelets adhered to the UBE sheet in a spread shape with pseudopods after 40 minutes, but there was no platelet aggregation (Figure 2, C). However, on the surface of the ePTFE sheets, aggregated platelets with fibrin were found after 40 minutes (Figure 2, D).

\section{Canine Study: Macroscopic Findings}

Table 2 shows the results of the macroscopic evaluation. At the time of re-sternotomy, 4 cases in group II showed adhesions between the sternum and the pericardium. The sheets of group I retained the implanted form (Figure 3, A), whereas the sheets of group II were crinkled, less pliable, and thickened by a dense layer of fibrous tissue (Figure 3, $B)$. The shrink ratio was significantly smaller in group I $(8.0 \%[5.5 \%, 12.4 \%]$ vs $31.7 \%$ [30.0\%, $44.8 \%] ; P=.006)$.

Group I showed no adhesions between the epicardium and the sheet and had no or minimal adhesions between the epicardium and the suture line (Figure 3,C). Two cases in group I showed a severe epicardial reaction, but in the remaining cases, the underlying structures were detectable. Conversely, group II showed minimum or moderate adhesions between the epicardium and the sheet and had moderate or severe adhesions between the epicardium and the suture line. In all cases in group II, the epicardial reaction was severe, and recognition of the underlying structures was completely impeded by fibrous tissue (Figure $3, D$ ).

\section{Microscopic Findings}

In group II, the surface of the epicardium beneath the synthetic sheet was covered with a thick and dense layer of collagenous fibrous tissue (stained blue in the Azan-Mallory- 

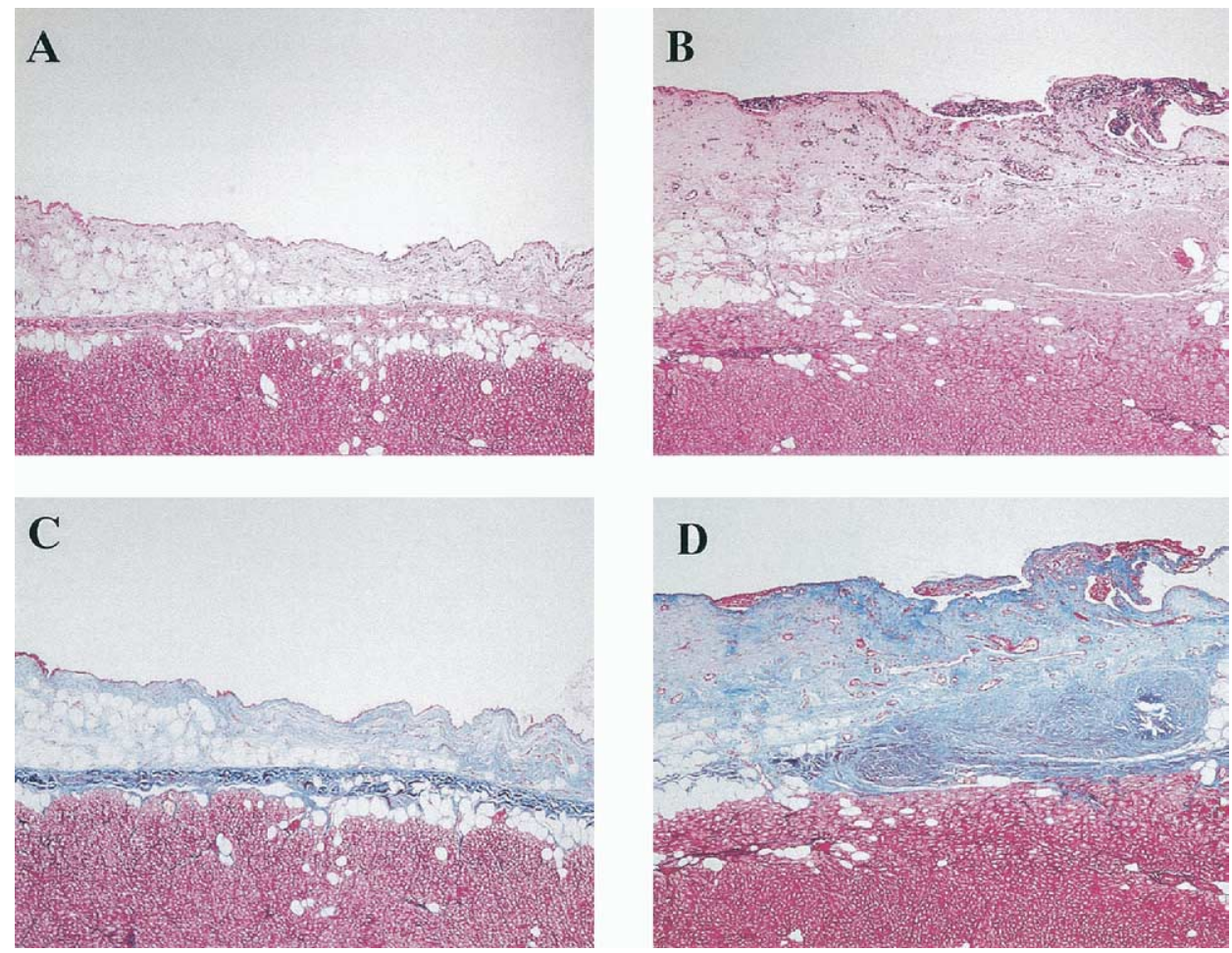

Figure 4. Cross sections through the tissue on the surface of the heart beneath the sheet (original magnification, $\times 40$ ). A, Group I, hematoxylin and eosin staining. B, Group II, hematoxylin and eosin staining. C, Group I, Azan-Mallory staining. D, Group II, Azan-Mallory staining.

stained section) with chronic active inflammatory reactions (Figure 4, B and D). Compared with group II, group I showed a thin layer of fibrous tissue covering the epicardium and mild chronic inflammatory reactions (Figure 4, A and $C$ ). The number of macrophages in the layer covering the epicardium was significantly less in group I (86 [56.8, $139.3]$ vs $201[161.0,276.5]$ in 3 fields; $P=.045)$ than in group II (Figure 5).

\section{Discussion}

Patients undergoing reoperation are at a higher operative risk than those undergoing initial procedures because of the formation of postoperative adhesions. ${ }^{1,2}$ In cardiac surgery, the importance of protecting the heart during repeat sternotomy has been indicated. ${ }^{19}$ Several biomaterials have been tried as pericardial substitutes, and the reported results have been mixed..$^{3-6,9,10}$ The ePTFE sheet, which is the most widely used synthetic pericardial substitute, was reported to develop tissue-free space around the synthetic sheet, which facilitates safe re-sternotomy. It also, however, brought on severe inflammatory reactions and diffuse fibrosis, and the sheet itself was deformed by a dense layer of fibrous tissue. ${ }^{4,10,20}$

We performed the platelet adhesion study to evaluate the blood compatibility of the sheets. Thrombogenicity of the sheets was evaluated by exposing the sheets to PRP. Less aggregate formation on the UBE sheet implied superior antithrombogenicity, which is currently recognized as a decisive factor in the development of antiadhesive membranes.

We selected a 3-layered structure-namely, a middle layer of polyester inserted between 2 layers of silicone polycarbonate polyurethane-to keep an implanted form of the pericardial substitute. Many investigators have reported that ePTFE sheets were deformed by a dense layer of fibrous tissue, although the sheets themselves did not seem shrunken or thickened. ${ }^{20}$ In this study, the ePTFE sheets were crinkled and thickened by a dense layer of fibrous tissue. However, the UBE sheets, consisting of 3 layers, retained their shape after implantation. In addition, there were no or minimal adhesions between the epicardium and the suture line of the UBE sheet. One possible explanation for the difference in suture-line adhesion may be the difference in size of the needle hole between the ePTFE and UBE sheets. This preliminary study, using scanned electron microscopy, showed that the hole size of the UBE sheet was smaller than the width of the needle, whereas the size of the needle hole of the ePTFE sheet was almost the same size as the width of the needle. These features may affect the results showing no or minimal adhesion between the 

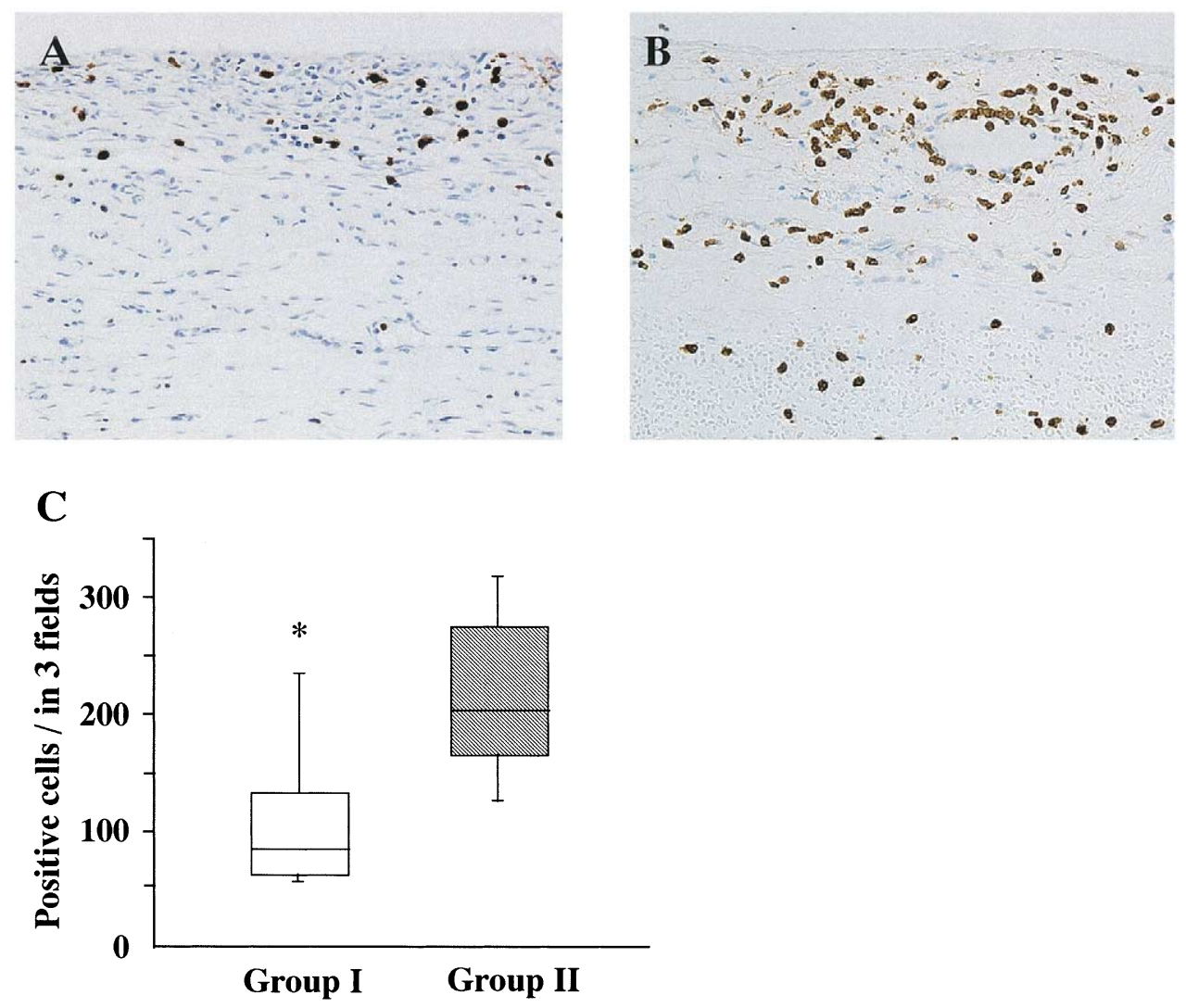

Figure 5. Macrophages in the layer covering the epicardium were stained with MAC387. A, In group I, a small number of macrophages existed. B, In group II, a large number of macrophages were recognized. C, The numbers of macrophages in the layer covering the epicardium are expressed as box and whisker plots. Horizontal lines in boxes denote 25th, 50th, and 75th percentile values from the bottom. The lower and upper whisker plots show minimum and maximum values, respectively. Group I shows fewer macrophages compared with group II. The number is the sum of the cell counts on 3 fields at $\times 400$ magnification. ${ }^{*} P=.045$ versus group II.

epicardium and the suture line in group I, because the fibrous tissue might not have been able to get into the holes.

The number of macrophages in the epicardial tissue was counted with immunohistochemical methods. Macrophages have been reported to play an important and direct role in adhesion formation..$^{21,22}$ Fibrosis, or adhesion formation, is an end phase in the inflammatory response. Macrophages secrete numerous mediators that in turn trigger the proliferation of fibroblasts. The mediators also trigger the synthesis and secretion of proteins that make up the extracellular matrix, such as collagen. ${ }^{21,22}$ Immunohistochemical findings showed that there were fewer macrophages in the covered epicardium in the UBE sheet group. We do not have an explanation for why the macrophage reaction to the UBE sheet was reduced. Because some cytokines that originate from platelets enhance the expression of monocyte chemoattractant protein, less thrombogenicity (less platelet aggregation) of the UBE sheet may account for the reduced macrophage (monocyte) reaction.
Limitations of this study should be noted. The experimental findings might not be completely reproducible clinically because the epicardial damage in this study was limited, cardiopulmonary bypass was not performed, and bleeding was minimal. In addition, although there was a distinct difference between groups, the follow-up period of 3 months is short; reoperations within a 3-month period are infrequent. Another concern was that in group I we had 2 cases with severe epicardial reactions. Although a minor infection or some kind of epicardial damage could be the cause, the reason for the reactions was unclear. Further experiments with longer observation periods are warranted. In addition, the development of new models to mimic clinical situations such as bleeding and epicardial damage is required.

In conclusion, the new 3-layered pericardial substitute that consists of silicone polycarbonate polyurethane outer layers and a middle layer of polyester significantly reduced adhesions after median sternotomy. This sheet may cause fewer adhesions and less severe epicardial reactions after 
cardiac surgery, thereby facilitating safe adhesiolysis during reoperation.

We express our gratitude to Tomomi Yamada, MS, for statistical analysis, and to the Department of Medical Information Science, Kyushu University Hospital.

\section{References}

1. Yau TM, Borger MA, Weisel RD, Ivanov J. The changing pattern of reoperative coronary surgery: trends in 1230 consecutive reoperations. J Thorac Cardiovasc Surg. 2000;120:156-63.

2. Loop FD. Catastrophic hemorrhage during sternal reentry. Ann Thorac Surg. 1984;37:271-2.

3. Bunton RW, Xabregas AA, Miller AP. Pericardial closure after cardiac operations. An animal study to assess currently available materials with particular reference to their suitability for use after coronary artery bypass grafting. J Thorac Cardiovasc Surg. 1990;100:99-107.

4. Revuelta JM, Garcia-Rinaldi R, Val F, Crego R, Duran CM. Expanded polytetrafluoroethylene surgical membrane for pericardial closure. An experimental study. J Thorac Cardiovasc Surg. 1985;89:451-5.

5. Gallo JI, Pomar JL, Artinano E, Val F, Duran CM. Heterologous pericardium for the closure of pericardial defects. Ann Thorac Surg. 1978;26:149-54

6. Heydorn WH, Daniel JS, Wade CE. A new look at pericardial substitutes. J Thorac Cardiovasc Surg. 1987;94:291-6.

7. Laks H, Hammond G, Geha AS. Use of silicone rubber as a pericardial substitute to facilitate reoperation in cardiac surgery. $J$ Thorac Cardiovasc Surg. 1981;82:88-92.

8. Malm T, Bowald S, Bylock A, Busch C. Prevention of postoperative pericardial adhesions by closure of the pericardium with absorbable polymer patches. An experimental study. J Thorac Cardiovasc Surg. 1992;104:600-7.

9. Mathisen SR, Wu HD, Sauvage LR, Walker MW. Prevention of retrosternal adhesions after pericardiotomy. J Thorac Cardiovasc Surg. 1986;92:92-8.
10. Meus PJ, Wernly JA, Campbell CD, Takanashi Y, Pick RL, Zhao-Kun Q, et al. Long-term evaluation of pericardial substitutes. J Thorac Cardiovasc Surg. 1983;85:54-8.

11. Phillips R, Frey M, Martin RO. Long-term performance of polyurethane pacing leads: mechanisms of design-related failures. Pacing Clin Electrophysiol. 1986;9:1166-72.

12. Zhao Q, Topham N, Anderson JM, Hiltner A, Lodoen G, Payet CR. Foreign-body giant cells and polyurethane biostability: in vivo correlation of cell adhesion and surface cracking. J Biomed Mater Res. 1991;25:177-83.

13. Takahara A, Coury AJ, Hergenrother RW, Cooper SL. Effect of soft segment chemistry on the biostability of segmented polyurethanes. I. In vitro oxidation. J Biomed Mater Res. 1991;25:341-56.

14. Reed AM, Potter J, Szycher M. A solution grade biostable polyurethane elastomer: ChronoFlex AR. J Biomater Appl. 1994;8:210-36.

15. Capone CD. Biostability of a non-ether polyurethane. J Biomater Appl. 1992;7:108-29.

16. Mathur AB, Collier TO, Kao WJ, Wiggins M, Schubert MA, Hiltner A, et al. In vivo biocompatibility and biostability of modified polyurethanes. J Biomed Mater Res. 1997;36:246-57.

17. Zhang Z, Roy R, Dugre FJ, Tessier D, Dao LH. In vitro biocompatibility study of electrically conductive polypyrrole-coated polyester fabrics. J Biomed Mater Res. 2001;57:63-71.

18. Ward RS. Thermoplastics silicone-urethane copolymers: a new class of biomedical elastomers. Med Device Diagn Industry. 2000;1:21-8.

19. Nkere UU, Whawell SA, Sarraf CE, Schofield JB, Thompson JN, Taylor KM. Perioperative histologic and ultrastructural changes in the pericardium and adhesions. Ann Thorac Surg. 1994;58:437-44.

20. Lu JH, Chang Y, Sung HW, Chiu YT, Yang PC, Hwang B. Heparinization on pericardial substitutes can reduce adhesion and epicardial inflammation in the dog. J Thorac Cardiovasc Surg. 1998;115:111120.

21. Kovacs EJ. Fibrogenic cytokines: the role of immune mediators in the development of scar tissue. Immunol Today. 1991;12:17-23.

22. Kovacs EJ, DiPietro LA. Fibrogenic cytokines and connective tissue production. FASEB J. 1994;8:854-61. 